\title{
EL ENFOQUE AL PRODUCTO Y EL ENFOQUE AL PROCESO: DOS METODOLOGÍAS COMPLEMENTARIAS PARA ENSEÑAR LA EXPRESIÓN ESCRITA EN LOS CURSOS DE UNA L2
}

Marta Madrigal Abarca

\section{(9) $\odot \Theta \Theta$}

Esta obra está bajo una licencia Creative Commons 



\title{
EL ENFOQUE AL PRODUCTO Y EL ENFOQUE AL PROCESO: DOS METODOLOGÍAS COMPLEMENTARIAS PARA ENSEÑAR LA EXPRESIÓN ESCRITA EN LOS CURSOS DE UNA L2
}

\author{
FOCUS ON THE PRODUCT AND FOCUS ON THE PROCESS: TWO \\ COMPLEMENTARY METHODOLOGIES TO TEACH WRITTEN \\ EXPRESSION IN L2 COURSES
}

Marta Madrigal Abarca

\begin{abstract}
RESUMEN
Para abordar la expresión escrita en una clase de español como segunda lengua existen diversos métodos que brindan una multiplicidad de estrategias compuestas de ejercicios y prácticas apropiados para construir textos cohesionados y coherentes. Este artículo expone los principios fundamentales del Enfoque al producto y el Enfoque al proceso con el fin de señalar los aspectos en los cuales ambas metodologías se imbrican y se complementan.

Palabras clave: expresión escrita, estrategias de escritura, enfoques cognitivos, el proceso de escritura, el enfoque al producto.
\end{abstract}

\begin{abstract}
To address the writing in a class of Spanish as second language, there are various methods that provide a multiplicity of strategies consisting of exercises and practices appropriate to build cohesive texts and consistent. This article sets out the fundamental principles of the approach to the product and the approach to the process in order to point out the aspects in which both methodologies are knit together and complement each other.

Key words: writing, strategies of writing, cognitive approaches, the writing process, the approach to the product.
\end{abstract}

\section{Introducción}

Generalmente, cuando se aborda el estudio de la enseñanza de lenguas siempre salen a la luz conceptos como competencias, estrategias y destrezas. Entre estas últimas se ubican la comprensión auditiva, la expresión oral, comprensión lectora y la expresión escrita. Sin embargo,

\footnotetext{
M. L. Marta Madrigal Abarca. Universidad de Costa Rica. Profesora de la Escuela de Filología y Lingüística. Costa Rica.

Correo electrónico: madrigal_ma@yahoo.com.mx
}

Recepción: 29- 04- 2015

Aceptación: 25- 08- 2015 
en la mayoría de los libros de texto y en los programas de lenguas se enfatizan el habla y la escucha, excluyendo las otras, pues, a veces, se piensa que solo puede haber comunicación cuando hay intercambio de palabras o frases de manera oral entre los interlocutores. Entonces, la enseñanza se reduce a estructuras gramaticales, vocabulario, morfología y sintaxis, porque, según esa idea, el dominio de estas habilidades da garantía de una comunicación eficaz.

Esa desestimación de la enseñanza de la expresión escrita se debe a distintas causas; algunos autores como Moreno, Moreno y Zurita (2009, p. 1) asocian esta falta de interés al auge del método comunicativo, el cual le dio más importancia a las otras destrezas, especialmente a las orales. Por su parte, Cassany $(2005$, p. 7) menciona que "el uso menor de la escritura se debe a sus características cualitativamente distintas", ya que el proceso de llevar a la forma escrita conceptos, pensamientos e ideas es complejo, pues el texto escrito representa el producto de una serie de operaciones mentales complicadas. Asimismo, los propios profesores relegan esta actividad de sus clases, porque requiere de mucho tiempo para revisar las producciones de sus estudiantes.

\subsection{Objetivo del trabajo}

Actualmente, existe una diversidad de metodologías para el desarrollo de la competencia escrita, las cuales se basan en teorías o puntos de vista sobre lo que es la lengua y cómo se aprende. El objetivo de este trabajo es señalar las principales nociones teóricas de los dos métodos comúnmente usados en las clases de español como L2 para la enseñanza de la escritura -el Enfoque al producto y el Enfoque al proceso- con la intención de mostrar los aportes de ambas metodologías, ya que son significativas por la incidencia que presentan en el análisis de las diversas propuestas didácticas de redacción que se han realizado a través de los años.

\section{Diferentes enfoques para la enseñanza de la escritura}

Los estudios sobre la expresión escrita tanto en lengua materna como en segundas lenguas han resaltado la complejidad del proceso de la escritura. Con el propósito de ahondar en esta destreza han aparecido diversas teorías y disciplinas provenientes de diferentes áreas de estudio que tratan de explicar y crear metodologías para su enseñanza; por ejemplo, las formulaciones de Van Dijk se relacionan con los estudios de lingüística textual y se basan en conceptos desarrollados en este campo. Las concepciones de Rohman (1965), las de Flower (1979) y Flower y Hayes (1981) están más próximas a la psicología cognitiva, mientras que las de Krashen (1982) están influidas por los estudios sobre los procesos de adquisición de una primera y segunda lengua.

Los distintos énfasis han ido cambiando a través del tiempo. Al principio, el interés de los investigadores se centraba en el texto mismo y en el análisis del sistema de la lengua. En los años ochenta, la tendencia apuntaba a las teorías cognitivas y psicológicas y privilegiaban los modelos de etapas y procesuales y, a partir de los años noventa, se experimenta una preferencia hacia los elementos sociales, contextuales y didácticos de la escritura (Arias-Fuentes, 2013, p. 35).

Toda esta diversidad de intereses ha generado una gran proliferación de métodos o enfoques que brindan estrategias diversas para la enseñanza de esta destreza, entre ellos están: el enfoque gramatical, cuyo propósito es enfatizar el conocimiento y el dominio de la gramática del sistema de la lengua; el enfoque funcional, que centra el aprendizaje a través de la comprensión y producción de los distintos tipos de textos escritos en situaciones específicas de uso, por tanto, la escritura se da con una finalidad ya sea informar, persuadir, opinar, etc.; el enfoque procesual, que se centra en los procesos cognitivos de composición que el aprendiz desarrolla como la generación de ideas y la manera en que se relacionan y se organizan dentro 
del texto; y el enfoque de contenido, el cual considera la escritura como parte del currículo y puede servir de instrumento para transmitir y obtener información de las materias del plan curricular. (Cassany, 1990, p. 1).

De estos, los enfoques más usados son los que enfatizan el producto y los que se centran en el proceso. El primero es más tradicional, pues considera que el fin último de la composición es un producto terminado, con correcciones en la estructura superficial del texto: ortografía, acentuación, palabras, oraciones, etc., obviando la estructura profunda (la organización lógico-semántica). El segundo ofrece un paradigma con el cual se explican, según Caldera (2003, p. 364): "los pasos o fases mentales que subyacen en la composición o producción de un texto". Es una metodología con una fuerte influencia de la psicología cognitiva, por tanto, desarrolla estrategias que toman en cuenta los procesos mentales que ocurren cuando se escribe.

\section{L Los modelos de composición cognitivos}

En estos modelos, el proceso de escritura se entiende como una actividad cognitiva compleja. Este criterio se debe a que el escritor, durante el trabajo de redacción, pone en funcionamiento una serie de estrategias y conocimientos, es decir, un conjunto de operaciones que interactúan entre sí para llegar a la construcción del texto. Se trata de explicar cuáles son los procesos que el escritor sigue durante el trabajo de redacción, pues el acto de escribir no solo es plasmar en un papel ideas o pensamientos, sino que "la escritura puede tener una función epistémica, lo que significa que puede contribuir a la elaboración del pensamiento" (Camps, 1990, p. 10).

La escritura es percibida como una actividad mediadora de los procesos psicológicos, por ello se accionan otras funciones cognitivas como la percepción, la atención, la memoria y el pensamiento. En consecuencia, el texto en sí es un sistema global que se construye paso a paso, a través de actividades cognitivas y subprocesos organizados (planificación o preparación, textualización o elaboración de borradores, revisión y edición) que son interactivos y recursivos, porque es posible hacer modificaciones al texto en cualquier etapa del proceso en la que se encuentre el escritor. Estas fases se ven también influidas por factores internos y externos como el conocimiento previo de las formas lingüísticas y del tema, el propósito, la audiencia y otras pautas del contexto comunicativo.

El modelo más influyente es el elaborado por Flower y Hayes (1981), el cual se explica con detalle tanto las estrategias que se utilizan para redactar como las operaciones intelectuales que conducen a la composición. Anteriormente, los investigadores se cuestionaron si el estudiante aprendía mediante el acto de la escritura y cómo se llevaba a cabo este aprendizaje. Sin embargo:

Flower y Hayes sostienen que analizando cómo el autor planifica el texto, cómo va regenerando sus objetivos, se pueden observar estos actos de aprendizaje en acción. Además, sugieren que la creatividad y la incentiva del escritor están estrechamente relacionadas con estos casos. (Cassany, 1989, p. 159)

Estos autores establecen, además, dos principios fundamentales: el de la memoria a largo plazo, que constituye un espacio donde el escritor guarda los conocimientos sobre el tema del texto, la audiencia y las distintas estructuras textuales que puede utilizar; y el proceso de escribir, el cual se compone de tres estadios: planificar, redactar y revisar, junto con un mecanismo de control denominado monitor que se encarga de regularlos y de decidir en qué momento actúa cada uno. La redacción se inicia cuando el escritor se enfrenta a una situación retórica: al problema o tarea que debe resolver. 
Otro criterio novedoso introducido por estos autores es la diferenciación hecha entre los escritores novatos y los expertos. Esta distinción estriba, básicamente, en la forma en que ambos tipos de escritores piensan y planifican su escrito pues, mientras los novatos hacen poca planificación (a veces nada), los segundos desarrollan estrategias retóricas donde establecen objetivos no solo de forma como de contenido para luego revisar globalmente todo el texto lo que da mayores probabilidades de obtener al final un producto mejor acabado.

\subsection{Los enfoques centrados en las fases de la escritura}

La manera de percibir la escritura como proceso favoreció la creación de diferentes metodologías que brindaron importantes aportes para la didáctica de esta estrategia. El modelo más destacado ha sido el de Flower y Hayes (1980), no obstante, según Cassany (1989), este ya había sido desarrollado por Gordon y Wlecke en 1964 y por G. Rohman en 1965. En estas propuestas o modelos de expresión escrita se establecían también tres etapas: pre-escribir, escribir y re-escribir. Pre-escribir engloba desde la necesidad del autor de escribir el texto hasta la obtención de la idea general y el plan o esquema. Seguidamente, el escribir y el re-escribir constituyen las etapas de propiamente de la redacción del escrito desde el esbozo de las primeras ideas hasta la corrección final o última. Se supone que siguiendo estas tres etapas ordenadamente se puede llegar a tener un texto aceptable con cohesión y coherencia. No obstante, este modelo fue muy criticado, ya que presentaba dos problemas. El primero es que, por un lado, suponía que las etapas se trabajaban en forma lineal, pues así consideraban el proceso de producción. Por otro lado, enfatizaba el producto final y no tomaba en cuenta los procesos internos del escritor; en otras palabras, no se interesaba en explicar qué pasaba por la mente de los escritores a la hora de redactar sus escritos.

Más tarde, las investigaciones posteriores determinaron que el proceso de composición de un texto no es lineal ni unidireccional, sino más bien recursivo (Beaugrande, 1982), es decir, no son pasos jerarquizados, sino que se usan cada vez que se necesiten, puesto que el escritor, cuando escribe, planea, busca ideas y las redacta, luego las revisa, etc. Esta recursividad se reduce a la aplicación de los subprocesos de planificación, textualización y revisión cuantas veces sea imprescindible. Al respecto, Cassany, Luna y Sanz (1994) afirman que "no existe un esquema lineal y lógico de trabajo, sino que cada persona, según su carácter, su estilo y también el problema retórico que se presenta actúa de una manera o de otra" (p. 267). Si el proceso de la redacción se desarrolla de manera completa y suficiente, el producto final será de calidad y esto es independiente del orden que se ha seguido en cada caso.

\subsection{El enfoque al proceso}

A través de este enfoque se enseñan los procesos cognitivos por los que atraviesa el escritor para elaborar su escrito; en otras palabras, se enfatizan las distintas actividades de pensamiento superior del autor desde la creación de una circunstancia social que exige producir un texto, hasta que este se dé por acabado. De esta manera, el texto final se percibe como el resultado de un proceso que involucra no solo los elementos que ocurren interiormente en la mente del escritor, sino también aquellos que proceden del exterior, esto es, las circunstancias que inducen o motivan a escribir. Se deja de pensar, entonces, que el texto final es un producto, un trabajo terminado sin discernir ni comprender cómo se llegó a él. 
La práctica de esta metodología en una clase de redacción supone un cambio de actitud tanto del docente como del estudiantado, pues los aprendices tienen que comprender que el acto de escribir no es solo rellenar con letras una hoja en blanco, sino que esta tarea implica una serie de actividades como el planeamiento del texto, la elaboración de esquemas, la redacción y revisión de borradores, el cotejo de las correcciones, entre otras.

Según Richards (1990, p. 108), estas actividades y estrategias cognitivas pueden ser determinadas empleando procedimientos como la observación, para averiguar cómo escriben los escritores; la entrevista antes y después de escribir, para establecer cómo negocian con aspectos particulares de una tarea de escritura; la verbalización, donde los escritores expresan las decisiones que ellos tomaron durante la escritura; y la revisión, que les permite examinar el diario de notas de sus procesos de escritura.

\subsubsection{Las tres etapas del Enfoque al proceso}

En este enfoque se diferencian tres etapas o fases llamadas preescritura, borrador y revisión. Es posible que el nombre de estas varíe según el investigador, pero su naturaleza es la misma; así por ejemplo, Calsamiglia y Tusón (2004, p. 82) distinguen tres estadios. El primero es la planificación, donde se ponen en juego la memoria y el contexto pragmático e incluye la definición de objetivos, la generación de ideas y su organización. Aquí se discriminan las informaciones relevantes de las irrelevantes, se jerarquizan las ideas y se ordenan, etc. Generalmente, las estrategias desarrolladas en esta fase se ubican en el inicio del proceso de la escritura; sin embargo, para Bereiter y Scardamalia (1987) se deben incluir durante todo el proceso y no solo al comenzar a escribir.

El segundo es la textualización que es la fase donde el escritor traduce los contenidos mentales en elementos de la lengua; en otras palabras, el autor toma decisiones en cuanto a la escogencia de las palabras adecuadas, al modo de conectar las frases entre sí, al uso de marcadores lingüísticos para dar cohesión a los enunciados y a la manera de construir párrafos. Además, toma en cuenta los aspectos morfosintácticos y las reglas ortográficas. El tercer estadio es la revisión. Implica las actividades retroactivas de lectura para evaluar el producto obtenido en el proceso de textualización.

El empleo de estas tres fases -las cuales no se desarrollan por separado, sino que se van complementando durante todo el proceso- da como resultado una comunicación escrita adecuada según el propósito: los escritores que utilizan de manera apropiada estas tres etapas obtienen un mejor control tanto en contenido como en la forma de lo que escriben (Richards, 1990, p. 109).

\subsubsection{El papel de los profesores y de los aprendices}

En esta forma de enseñanza, el papel de los aprendices y de los profesores es muy característico, ya que el énfasis se dirige al estudiante y no al texto que se va a redactar. Se concibe al escritor como único e individual de manera que debe desarrollar sus propias estrategias de acuerdo con sus habilidades y su personalidad. Cada estudiante tiene que encontrar su propio estilo de trabajo, el cual le permita seleccionar las técnicas más productivas e integrarlas a su propio trabajo de composición. Debe asumir un control mayor sobre lo que escribe, en cómo lo hace y afrontar con madurez tanto sus propias correcciones como las evaluaciones que hagan sus compañeros de su escrito. 
El papel del profesor también es redefinido y negociado en tanto que se convierte en un investigador de los procesos de escritura que emplean sus estudiantes. Por medio de la observación y de la discusión, el educador puede identificar acercamientos más exitosos en las diferentes fases del proceso para sus alumnos. En otras palabras, la idea no es obligar a los aprendices a producir textos correctos, sino abandonar el rol tradicional y unidireccional que solo propone modelos a seguir, para actuar como facilitadores, organizando experiencias de escritura que le permitan al aprendiz desarrollar estrategias de composición efectivas. Como lo señala Brookes y Grundy (1998, p. 15) su trabajo es guiar, en todo momento, al aprendiz en la búsqueda de ideas, en la planeación de estructuras, en la elaboración de múltiples borradores; ayudarlos para que tomen conciencia de sus debilidades y de sus puntos fuertes, sugiriéndoles las técnicas adecuadas de acuerdo a posibilidades. De manera que:

\footnotetext{
El rol del profesor es ayudar al estudiante a desarrollar estrategias viables para poder empezar (encontrando temas, generando ideas e información, enfocando y planeando estructuras y procedimientos), para esbozar (motivando múltiples borradores), para revisar (agregando, eliminando, modificando, arreglando ideas) y para editar (atendiendo el vocabulario, la estructura de la frase gramatical y la mecánica). (Cassany, 1990, p. 16)
}

Se trata de un trabajo muy individualizado con el sujeto de la escritura y no con el objeto, es decir, con el texto.

\subsubsection{Tareas y ejercicios en las tres etapas}

Las clases basadas en el proceso adoptan diversas formas de trabajo, ya sea por medio de tareas programadas, con ejercicios planeados con instrucciones precisas y puntuales sobre lo que se tiene que hacer, o de manera espontánea según el ritmo y los intereses de cada estudiante, de forma que las tareas sean más generales y abiertas. Las autoras Figueras y Santiago (2000, pp. 15-16) proponen diversas estrategias para trabajar el proceso global de composición. Para ellas, primero se debe analizar la situación comunicativa, en otras palabras, se trata de responder preguntas como: ¿quién leerá el escrito?, ¿qué se quiere conseguir?, ¿qué se sabe del tema?, ¿qué es necesario averiguar sobre el tema?, ¿cómo se organizará la información?, ¿qué tipo de secuencias textuales se emplearán? Además, se debe determinar cómo será el texto (extensión, tono, presentación, etc.). En esta primera fase, la preescritura, los ejercicios son diseñados para ayudar a esbozar o esquematizar lo que será la redacción. Entre estos se encuentra: la lluvia de ideas, los diarios, los mapas de palabras, los ideogramas, esquemas, la asociación libre, la escritura rápida y otras actividades que le sirvan al estudiante a reunir información, por ejemplo, lecturas diversas, canciones, etc.

En la segunda fase, el borrador o textualización, el estudiante se dedica al intento inicial de elaborar el bosquejo de las diferentes secciones del escrito. Es el tiempo para esquematizar el problema retórico. Luego de obtener toda la información necesaria, se empieza a escribir el primer borrador siguiendo el esquema trazado, esto es, se procede a plasmar sobre el papel las ideas, a concentrarse selectivamente en diversos aspectos del texto. Aquí, el escritor se enfoca en el manejo del lenguaje, utilizando la sintaxis correcta y seleccionando el vocabulario apropiado. Además, se pueden incluir ejercicios como párrafos desordenados, redacción de tesis y frase tópica, escritura rápida, borrador en grupo, etc.

Finalmente, en la fase de la revisión, el estudiante se dedica a editar su escrito tanto en contenido como en forma, a comparar el texto producido con los planes previos, a retocar el texto eliminando o añadiendo palabras o frases, utilizando sinónimos, entre otros. Según lo mencionan 
Cassany, Luna, y Sanz, (1994): “el autor compara el escrito realizado en aquel momento con los objetivos planificados previamente y lo retoca para adaptarse a ellos y mejorarlo" (p. 267). Es posible utilizar el chequeo por medio de listas que contienen nociones sobre ortografía, acentuación, puntuación y conocimientos gramaticales; ejercitar la reescritura de frases y párrafos y, además, acentuar la retroalimentación, tanto entre los estudiantes como con el profesor.

\subsubsection{La evaluación}

En esta metodología, la evaluación también debe ser rediseñada, ya que se trata de valorar cómo el estudiante elabora su redacción tomando en cuenta las estrategias que emplea, las técnicas, el proceso cognitivo, etc. Se enfatiza especialmente la fórmula guía-cooperación, donde la responsabilidad queda más repartida, porque el estudiante en la etapa de revisión tiene la oportunidad de autocorregirse y de reescribir o reformular su escrito. Esta manera de revisar en colaboración mutua es la única que restituye la autoridad del auténtico autor del texto: el alumno.

Por otra parte, como se valora un proceso dinámico y cambiante se utiliza una evaluación cualitativa, basada en la observación y en la recogida de datos. Para ello:

Se hacen entrevistas a los alumnos, se los observa mientras escriben, se analizan comparativamente los borradores que producen, etc. Más que poner una nota numérica a cada alumno, lo que interesa es descubrir tendencias, estilos de trabajo y progresos en el proceso de composición de cada individuo.

(Cassany, Luna y Sanz, 1994, p. 297)

Se puede decir con propiedad que se emplea una evaluación formativa, la cual constituye un instrumento facilitador del proceso de revisión, utilizado durante el proceso de escritura, ya que, según la autora Ribas y Seix (1993, p. 30): “proporciona al alumno (y también al profesor) la información para valorar el propio texto en curso de elaboración y tomar decisiones para mejorarlo en un sentido concreto".

En el siguiente cuadro se exponen las pautas que podrían ser utilizadas para observar y medir, de alguna manera, el trabajo de los estudiantes.

\section{Cuadro 1.}

\begin{tabular}{|c|c|}
\hline & Actividades \\
\hline $1^{\circ}$ Fase: preescritura & $\begin{array}{l}\text { - } \quad \text { Busca y desarrolla ideas } \\
\text { - Usa diferentes técnicas para generar ideas } \\
\text { - Anota las ideas con palabras y expresiones que } \\
\text { entiendan los lectores } \\
\text { - Anota las ideas para sí mismo y luego las explica } \\
\text { - Considera la audiencia y el propósito }\end{array}$ \\
\hline $2^{\circ}$ Fase: borrador & $\begin{array}{ll}\text { - } & \text { Elabora planes o esquemas } \\
\text { - } & \text { Escribe más de un borrador } \\
\text { - } & \text { Selecciona la información pertinente } \\
\text { - } & \text { Organiza el texto en párrafos } \\
\text { - } & \text { Estructura los datos en forma lógica } \\
\text { - } & \text { Utiliza oraciones temáticas } \\
\text { - } & \text { Emplea correctamente los signos de puntuación }\end{array}$ \\
\hline $3^{\circ}$ Fase: revisión & $\begin{array}{ll}\text { - } & \text { Revisa tanto la forma como el contenido } \\
\text { - } & \text { Usa pautas de corrección } \\
\text { - } & \text { Interactúa con los compañeros } \\
\text { - } & \text { Acepta las correcciones de los compañeros } \\
\text { - } & \text { Acepta las correcciones del profesor } \\
\end{array}$ \\
\hline
\end{tabular}


En resumen, el tiempo de la clase es un espacio libre y autónomo, donde cada alumno trabaja a su propio ritmo. Según Cassany (1990, p. 76), son clases más parecidas a un taller de escritura, aunque lo que escriban los estudiantes no necesariamente sean textos literarios o con intención artística o lúdica, sino cartas, ensayos trabajos escolares, textos académicos, entre otros.

\section{El enfoque al producto}

Los métodos centrados en el producto se centran en el resultado de la escritura, es decir, en el texto mismo. Su principal finalidad es el desarrollo de la competencia textual desde una perspectiva principalmente lingüística; por ejemplo, el enfoque basado en la enseñanza de la gramática, el cual supone que el dominio de la morfosintaxis, la ortografía y el léxico es el requisito para componer un texto, y el enfoque basado en funciones o enfoque didácticotextual, el cual se basa en el enfoque nociofuncional de la enseñanza de lenguas y en la tipología de textos.

En general, las metodologías usadas en las clases de escritura que enfatizan el producto final consideran la expresión escrita como el fin de la enseñanza, a diferencia de los modelos por etapas que promueven la escritura como una forma de instruir y ejercitar no solo aspectos gramaticales, sino procesos y estrategias integradas en actividades y ejercicios que donde se empleen todas las destrezas.

Richards (1990, p. 106) propone los principales rasgos del Enfoque al producto de la siguiente manera:

- Se redactan textos específicos que los aprendices requieren, ya sea escritura institucional o personal.

- Se enfoca en patrones y formas de organización de diferentes secuencias textuales como narración, descripción, exposición argumentación para ser usados en redacción de ensayos o reportes.

- Se presentan las reglas gramaticales en composiciones modelos, es decir, textos que sirvan de ejemplos para que los aprendices usen en sus escritos.

- Se corrigen oraciones; se da énfasis a las reglas gramaticales.

- Se le brinda al estudiante modelos o guías a seguir y controles para evitar cometer errores; se enseñan aspectos como caligrafía, ortografía, puntuación, deletreo.

Puede decirse, entonces, que este enfoque presenta dos cometidos primordiales: por un lado, proveer la práctica adecuada para producir diferentes clases de textos y, por otro lado, evitar que los estudiantes cometan errores. En esto se asemeja al enfoque gramatical descrito, ya que la expresión escrita se identifica con la gramática, específicamente la ortografía y la sintaxis, por ello se dice que está relacionado con los estudios normativos y estructuralistas de gramática, de larga tradición pedagógica que "la gran mayoría de maestros y alumnos hemos heredado de la escuela" (Cassany, Luna y Sanz, 1994, p. 273).

\subsection{Actividades y tareas}

Generalmente, las técnicas usadas en el enfoque al producto empiezan con ejercicios de escritura controlada y pasan, de manera gradual, hacia una escritura más libre cuando ya se ha memorizado la estructura que se trabajó. Las actividades incluyen ejercicios de sustitución, construcción de párrafos a partir de cuadros, tablas u otras guías, producción del texto a partir de respuestas a un grupo de preguntas, el desarrollo de oraciones complejas siguiendo diferentes 
reglas de combinación. A través de estas prácticas, el aprendiz va adquiriendo y desarrollando cada día más la habilidad de escribir, de manera que va elaborando tareas más complejas; usa una gran variedad de modos retóricos y discursivos; además, domina más aspectos complejos del párrafo y de la organización de oraciones.

A pesar de los valiosos resultados obtenidos al abordar la escritura desde el enfoque al producto, los investigadores critican que el interés se concentra primordialmente en el fin y no en los estadios medios. Se le da más énfasis a la forma y a la estructura que a las estrategias que los escritores usan para crear sus composiciones. En resumen, es un modelo que favorece más el producto terminado que el proceso mediante el cual se produjo el texto.

\subsection{El papel de los profesores y de los estudiantes}

Por su parte, el papel de los profesores y de los estudiantes tiende a conservar los rasgos tradicionales de la enseñanza, a saber, una instrucción unidireccional donde los alumnos realizan las tareas y ejercicios que se les impone. De manera que el maestro decide qué, cómo y cuándo se escribe y el estudiante solamente sigue esas instrucciones. Además, mientras el docente se apropia de la responsabilidad de corregir todos los ejercicios, el estudiante no está capacitado para autocorregirse o corregir a un compañero y siempre espera que esta tarea la realice el profesor, ya que la escritura sin corrección pierde sentido.

En esta perspectiva de trabajo, el profesor debe enmendar todos los errores del texto hasta quedar perfecto y el estudiante no puede cometer errores, porque crean hábito. Es así como, en este juego de roles, el alumno asume muy poca responsabilidad en el proceso global de trabajo; pronto se da cuenta de que el maestro es quien decide sobre todo el ejercicio de escritura e ignoran, ambos, las estrategias y técnicas mediante las cuales se llegó a una redacción final que se supone esté perfectamente acabada.

\subsection{La evaluación}

En este método, el estudiante, rara vez, tiene la oportunidad de revisar y analizar sus propios errores, de autocorregirse, de reformular borradores y mejorar así su escrito. Por lo general, la evaluación del producto que más se usa es la prueba donde el alumno tiene que redactar un texto. Sin embargo, para que esta sea válida y fiable debe cumplir con ciertos requisitos evaluativos tales como los descritos por Cassany, Luna y Sanz (1994, pp. 292-293) a continuación:

\footnotetext{
1. Objetivos: selección de ejes que respondan a los contenidos del curso y a los intereses de la evaluación para elaborar la prueba que se adecue a ellos como el dominio de la ortografía y de la normativa escrita de la lengua, el uso adecuado de la variable estándar y los registros formales, conocimientos de la estructura de los textos, elaboración de resúmenes, dominio de los signos de puntuación y acentuación, grado de madurez sintáctica.

2. Ejercicio y tipo de texto de la prueba: con los objetivos escogidos y formulados se selecciona el ejercicio conveniente, por ejemplo: dictado, llenar espacios en blanco, hacer un resumen, redactar un tipo de texto, etc.

3. Criterios de éxito y baremos: aquí se incluyen las pautas que se aplicarán en la corrección, es decir, el valor numérico de cada aspecto.

4. Sistemas de corrección: estas son bandas analíticas que repasan punto por punto el baremo y van restando los aspectos deficientes.
}

Existen muchas formas de confeccionar un baremo, esto va a depender de los objetivos y de los criterios que el profesor necesite evaluar. Un ejemplo puede ser el siguiente: 
Cuadro 2.

\begin{tabular}{|l|l|}
\hline I. CONTENIDO & PUNTAJE \\
\hline 1. El tema está bien definido y limitado & \\
\hline 2. El problema y la tesis están bien planteados & \\
\hline 3. Se reconocen las posturas y perspectivas & \\
\hline 4. Los puntos de vista son consistentes & \\
\hline 5. Las ideas expresadas se relacionan con el título & \\
\hline 6. Se utiliza el registro adecuado: estándar y formal & \\
\hline II. ORGANIZACIÓN & \\
\hline 7. Hay secuencia de ideas & \\
\hline 8. El texto tiene la estructura solicitada & \\
\hline 9. Hay variedad de ideas relacionadas y complementarias & \\
\hline 10. Los párrafos están bien estructurados & \\
\hline 11. Se plantea la introducción, el desarrollo y la conclusión & \\
\hline III. EXPRESIÓN & \\
\hline 12. Usa el vocabulario apropiado con el tema & \\
\hline 13. Ausencia de vocablos extranjeros, palabras inventadas o de sentido impropio & \\
\hline 14. Evita la repetición de palabras y la cacofonía & \\
\hline 15. Usa conectores que dan cohesión al texto & \\
\hline IV. GRAMÁTICA & \\
\hline 16. Usa la conjugación apropiada y la concordancia de tiempos & \\
\hline 17. Hay concordancia de género y número & \\
\hline 18. Usa adecuadamente los gerundios y los participios & \\
\hline V. NOTACIÓN & \\
\hline 19. Usa los signos de puntuación requeridos & \\
\hline 20. Acentúa correctamente & \\
\hline 21. Usa de manera apropiada las reglas ortográficas & \\
\hline
\end{tabular}

Para obtener la calificación final, a cada criterio se le asigna un puntaje de esta forma: 5 (Excelente), 4 (Muy bueno), 3 (Bueno), 2 (Regular), 1 (Deficiente). El puntaje total se obtiene de la multiplicación del número de puntos obtenidos x 100 divido entre el total de puntos de la escala empleada.

\section{Dos perspectivas complementarias}

Tanto el enfoque al producto como el enfoque al proceso tienen características muy definidas y criterios muy específicos en cómo enfrentar la enseñanza de la escritura. Ambos son válidos, porque los resultados que se alcanzan al ejecutar una clase con cualquiera de los 
dos son importantes, pues siempre hay un mejoramiento en la redacción. No obstante, aunque cada uno tenga rasgos muy específicos, no necesariamente son excluyentes, es decir, que se pueden tomar las estrategias y actividades de un enfoque para adaptarlas al otro y esto no perjudicaría, en ningún aspecto, los objetivos trazados en el curso.

Asimismo, ambas posturas van en busca de un resultado exitoso, esto es, de una redacción cohesionada y coherente, la diferencia está en la manera en que se utilizan las estrategias y en cómo se abordan los ejercicios y las actividades. Las estrategias didácticas constituyen excelentes herramientas para desarrollar el pensamiento crítico y creativo de los estudiantes mientras aprenden. Por ello, ¿por qué no aprovecharlas sea cual sea el enfoque que se esté empleando? Por ejemplo, muchas actividades son quizá muy valiosas, pero si son realizadas de forma individual, no les ayudarán a los estudiantes a enriquecerse con las aportaciones y experiencias de los compañeros como ocurriría si se incorporara a la clase, la práctica habitual de la retroalimentación.

\section{Consideraciones finales}

Actualmente, la expresión escrita se considera una manifestación lingüística con características propias que requiere de modelos específicos para su enseñanza y aprendizaje. La percepción de la escritura, como una actividad cognitiva compleja, ha motivado la creación de muy variados estilos o métodos; sin embargo, estos no presentan una uniformidad de criterios para decidir cuál es el más conveniente o cuál se ajusta más a los propósitos que se tienen para programar una clase de redacción. Por ello, los investigadores se debaten en la polémica de cuál de todos es el más eficaz.

Ese es el caso de los dos enfoques a los cuales se hizo referencia en este artículo: el enfoque al producto y el enfoque al proceso. Algunos profesores prefieren trabajar con los estudiantes en búsqueda de una redacción final, la cual deberá ser revisada repetidamente hasta llegar a ser un texto aceptable, sin poner atención en lo interesante y efectivo que puede resultar el proceso por medio del cual se logró ese producto (Brookes y Grundy, 1998, p. 15). Otros eligen un planteamiento más creativo y abierto, donde los estudiantes vayan tomando conciencia de sus habilidades y destrezas paso a paso. De acuerdo con Camps (1997), los cursos que se centran en el proceso parecen ser más efectivos que los centrados en el producto, porque permite a los aprendices explorar un acercamiento a una práctica de escritura más personal, mientras que en los cursos en los que se enfoca el producto como tal, las correcciones se habían mostrado altamente ineficaces.

Por su parte, Jurado-Spuch (2005) impulsa un acercamiento a los dos métodos y expresa que "both process and product should equally be taken into account when designing writing activities" (p. 669). Pero, la práctica ha demostrado que no existe un enfoque único y verdadero, sino que este deberá estar determinado por varios factores como el nivel de los estudiantes, la situación en la que estén aprendiendo la lengua (sea lengua materna o lengua extranjera) y las metas y las necesidades que presenten los alumnos. Esta condición conmina al docente a evaluar, de manera razonada y profesional, las diferentes posturas o enfoques, preservando los puntos de vista que le sean útiles en sus clases. Es un reto que el profesorado actual debe enfrentar para un mejor conocimiento y dominio de su trabajo. Según Richards y Theodore (1998, p. 7) esta invención de nuevas prácticas y enfoques para elaborar programas de lengua y materiales refleja un compromiso para encontrar formas más eficaces y eficientes de enseñar lenguas y, en este caso, enseñar expresión escrita. 
De todas formas, una clase de redacción no es un espacio cerrado y homogéneo cuyos participantes tienen las mismas características que puedan ser encapsuladas en moldes o modelos preestablecidos. Contrariamente, cada estudiante es un individuo particular, con diferentes habilidades y formas de aprender; por tanto, las clases deben adecuarse a la población con la que se vaya a trabajar. Parafraseando a Richards (1990, p. 114), un profesor de escritura exitoso no es el que ha desarrollado un método, sino el que ha creado un ambiente efectivo donde los aprendices se sientan cómodos descubriendo sus propias fortalezas y debilidades como escritores.

\section{Bibliografía}

Arias-Fuentes, D. (2013) La escritura como proceso, como producto, como objetivo didáctico. Tinkuy: Boletín de investigación y debate. 19, 33-46. http://dialnet.unirioja.es/servlet/ oaiart?codigo $=4736534$ [Consulta 20 de enero de 2015].

Beaugrande, R. De. (1982). Psychology and composition: past, present, and future. Por M. Nystrand (Ed.). What writers know. (211-267). Nueva York: Academic Press.

Bereiter, C. y Scardamalia, M. (1987). The Psychology of Written Composition. Londres: Earlbaum.

Brookes, A. y Grundy, P. (1998). Beginning to write: writing activities for elementary and intermediate learners. Cambridge: Cambridge University Press.

Calsamiglia, H. y Tusón, A. (2004). Las cosas del decir. Barcelona: Editorial Ariel.

Caldera, R. (2003). El Enfoque Cognitivo de la escritura y sus consecuencias. www.redalyc. org/articulo.oa?id=35662002 [Consulta 1 de febrero de 2015].

Camps, A. (1990). Modelos del proceso de redacción: algunas implicaciones para su enseñanza. Infancia y Aprendizaje.49,3-19. http://DialnetModelosDelProcesoDeRedaccion-48341. pdf [Consulta 6 de febrero de 2015].

Camps, A. (1997). Escribir. La enseñanza y el aprendizaje de la composición escrita. Signos. Teoría y práctica de la educación. 20, 24-33. upvv.clavijero.edu.mx/cursos/.../vector2/ actividad9 [Consulta 6 de febrero de 2015].

Cassany, D. (1989). Describir el escribir. Barcelona: Ediciones Paidós.

Cassany, D. (1990). Enfoques didácticos para la enseñanza de la expresión escrita. Comunicación, lenguaje y educación. 6, 63-80.

Cassany, D., Luna, M. y Sanz, G. (1994). Enseñar lengua. Barcelona: Editorial Graó.

Cassany, D. (2005). Expresión escrita en L2/ELE. Madrid: Arco Libros.

Figueras, C. y Santiago, M. (2000). Planificación. Por E. Montolío (Comp.) Manual práctico de escritura académica. (Vol. 2). (15-68). Barcelona: Editorial Ariel.

Flower, L. y Hayes, J. (1981). A Cognitive Process. Theory of Writing. College Composition and Communication. 32 (4), 365-387.

Flower, L. (1979). Writer-Based-Prose: A Cognitive Basis for Problems in Writing. College English. 41 (1), 19-37. 
Jurado-Spuch, A. (2005). Writing activities should be as much concerned with process as With product. Actas II, IX Simposio Internacional de Comunicación Social. Centro De Lingüística Aplicada. Santiago de Cuba. (669-673). Santiago de Cuba: Centro de Lingüística Aplicada.

Krashen, S. (1982). Principle and Practice in Second Language Acquisition. http://www. sdkrashen.com/Principles_and_Practice/Principles_and_Practice.pdf [Consulta el 16 de diciembre de 2014].

Moreno, C., Moreno, V. y Zurita, P. (2009). A escribir se aprende escribiendo. Marco ELE. Revista de didáctica español como segunda lengua. 8. http://marcoele.com/ descargas/8/moreno_moreno_zurita.pdf [Consulta 22 de enero de 2015].

Ribas y Seix, T. (1993). La evaluación de la composición escrita. Cuadernos de Pedagogía. 216, 28-30.

Richards, J. (1990). The Language Matrix. Cambridge: Cambridge University.

Richards, J. y Theodore, R. (1998). Enfoques y métodos en la enseñanza de idiomas. Cambridge: Cambridge University Press.

Rohman, G. (1965). Pre-Writing: The stage of Discovery in the Writing Process. College Composition and Communication. 16 (2), 106-112. 
